\title{
RESPUESTA DEL CULTIVO DE MAÍZ A LA LABRANZA DE CONSERVACIÓN EN CUATRO DIFERENTES SUELOS TROPICALES1
}

\author{
Jesús Ureste ${ }^{2}$, Santos Campos $^{2}$
}

\begin{abstract}
RESUMEN
Respuesta del cultivo de maíz a la labranza de conservación en cuatro diferentes suelos tropicales. Bajo condiciones de temporal. en 1993 se evaluaron en la zona centro del estado de Vcracruz, México, la respuesta del maíz a los sistemas de labranza convencional. reducida y cero en cuatro suelos tropicales con diferentes textura y grado de drenaje. Las variables medidas fueron densidad de población y rendimiento de grano. El aspecto económico se midió con la rclación Beneficio/Costo. En los suelos Fluvisoles de textura fina y media drenados, se obtuvo la mayor y más uniforme densidad de población, así como el mayor rendimiento de grano, no existiendo practicamente diferencia entre estos dos suelos y entre los cuatro sistemas de labranza. En el sucio Luvisol de textura gruesa muy drenado, la densidad de población fue mayor en cero labranza que en la labranza reducida y convencional. sin embargo, el rendimiento de grano obtenido con labranza reducida y cero fue prácticamente igual entre sí, pero superior al obtenido con la labranza convencional. En el suelo Vertisol arcilloso mal drenado se obtuvo el menor rendimiento de grano con los cuatro sistemas de labranza y éste fue mayor en labranza convencional que en la labranza cero y reducida. Con los sistemas de labranza cero y reducida se obtuvo la mayor relación Beneficio/Costo en los sucios Fluvisoles y Luvisolcs. En el suelo Vertisol arcilloso mal drenado no es posible producir maíz económicamente con cualquiera de los sistemas de labranza estudiados, a menos que se utilice alguna práctica para reducir el exceso de humedad del sucio.
\end{abstract}

\begin{abstract}
Corn (Zea mayz L.) response to conservation tillage on four tropical soils. Under the rainfed conditions of the central zonc of Veracruz Mexico, the res pon se of the maize crop to the conventional, reduced and zero tillage systems was evaluated in four tropical soils of different texture and drainagc degree. Plant population and grain yield were used as indicating variables. Economical aspects were measured by the Cost/Benefit relationship. The most uniform and highest plant population and grain yield were achieved in the fine and medium textured, well drained Fluvisols, showing no difference between these two soils or among the four tillage systems. In the coarse textured, excessively drained Luvisol, the plant population was higher with zero tillage than with the reduced and conventional tillage; however, the grain yield achieved with zero and reduced tillage was practically alike, but higher than that achieved with the conventional tillage. In the fine textured, poorly drained Venisol, the lowest graio yield was obtained with the four tillage systems, being higher for the conventional tillage system. Zero and reduced tillage reached the highest Cost/Benefit relationship in the Fluvisols and Luvisols. On the clay Vertisol soils, it is not economically feasible to produce corn with any of the studied tillage systems, unless the excess moisture in the soil is reduced.
\end{abstract}

\section{INTRODUCCIÓN}

Actualmente, en el estado de Veracruz, México, la mayoría de los agricultores preparan la cama de siembra para el cullivo de maíz en forma mecánica, ya sea utilizando tracción motriz o animal. Estas labores realizadas muchas veces en forma excesiva, consisten generalmente en barbecho (volteo del suelo) más rastreo y surcado.

Aunque no existe documentación suficiente para apoyar su uso, estas labores, se recomiendan

\footnotetext{
1 Presentado en la XL Reunión Anual del PCCMCA en Costa Rica, América Central. 13 al 19 de marzo, 1994.

2 INIFAP-Campo Experimental Cotaxtla. Ap. Postal 429, CP. 91700 Veracruz, México. Teléfono (29)34-8343. Fax (29)34-8591.
} 
indiscriminadamente sin importar tipo de cultivo y suelo, lo cual al realizarse en condiciones no óptimas de humedad, topografía, etc., contribuyen a la erosión y degradación del suelo con la subsecuente redución del rendimiento del maíz. Desde el punto de vista económico, la preparación de la cama de siembra en forma convencional. para el cultivo de maíz utiliza como mínimo el $25 \%$ del costo total de producción, el cual es alto al compararlo con un costo de 14 y 8 porciento bajo los sistemas de mínima y cero labranza respectivamente.

Los sistemas de labranza de conservación (labranza reducida y cero), ofrecen varias ventajas al compararlos con la labranza convencional, entre las cuales sobresalen la reducción hasta en $90 \%$ de la pérdida de suelo (Uresti y Cornish, 1986), mayor humedad disponible para los cultivos (Phillips, 1974 y Phillips et al. 1980),25\% de reducción de los costos de producción (Uresti y Cadena 1994) y obtención de rendimientos iguales o superiores que en la labranza convencional (Wendt y Burwell, 1985).

Debido a estas ventajas, a partir de la década pasada estos sistemas de labranza de conservación están siendo recomendadas y adoptados rápidamente por los productores de maíz en las zonas tropicales de Veracruz.

Sin embargo, existen evidencias obtenidas principalmente en zonas de clima templado que indican que los sistemas de labranza de conservación no son óptimos para producir maíz en algunos tipos de suelo como los arcillosos pobremente drenados, donde el exceso de humedad disminuye el rendimiento de grano (Phillips et al. 1980 y Cosper, 1983).

Investigaciones recientes muestran que a mediano y largo plazo el sistema de labranza cero en particular puede tener un efecto negativo en algunas propiedades y características físico-químicas del suelo, afectando por lo tanto el rendimiento de grano de maíz.

En el trabajo realizado por Rhotan et al. (1983), en suelos de textura franco arenosa, franco, limoso y franco arcillo-arenoso, encontraron un incremento en la acidez y la densidad aparente del suelo bajo cero labranza, sin embargo, indican que este incremento no fue significativo al compararlo con la labranza convencional.

Franzen et al. (1994), encontraron que en suelos de textura arenosa bajo labranza cero ocurrió una severa compactación de la capa superficial al incrementarse la densidad aparente y resistencia a la penetración. Resultados similares fueron obtenidos por Uresti y Cadena (1994), al concluir que después de cuatro años de producir maíz bajo los sistemas de labranza convencional y cero en un suelo migajon-arenoso de la zona central de Veracruz, la densidad aparente promedio de los $20 \mathrm{~cm}$. superiores del suelo fue de $1,52 \mathrm{~g} / \mathrm{cc}$ y $1,73 \mathrm{~g} / \mathrm{cc}$ respectivamente,.indicando que el menor rendimiento obtenidos en cero labranza pudo deberse en parte a este incremento de la densidad aparente y compactación del suelo.

En suelos francos y franco limosos, Vyn et al. (1982) encontraron que la densidad aparente y resistencia a la penetración del suelo se incrementaron en cero labranza al compararlos con la labranza convencional, sin embargo, indican que estos cambios no se asocian significativamente con la reducción de $10 \%$ en rendimiento de grano de maíz obtenido bajo cero labranza.

Ante estas evidencias, la recomendación indiscriminada de los sistemas de labranza de conservación puede resultar contraproducente, por lo que es necesario realizar investigaciones con el fin de definir recomendaciones específicas de labranza que no afecten negativamente las características del suelo y el rendimiento de grano de maíz, o en su caso, plantear soluciones alternativas a los problemas encontrados.

Dentro de este contexto se planteó el presente trabajo programado a cinco años, para evaluar el efecto de cuatro intensidades de labranza en cuatro suelos tropicales con diferente textura y grado de drenaje, sobre el rendimiento de grano de maíz y las características del suelo. Los resultados que se presentan en este artículo son un avance y corresponden al primer año de la evaluación. 


\section{Objetivos}

Evaluar el efecto de cuatro intensidades de labranza en cuatro suelos tropicales con diferente textura y grado de drenaje sobre el rendimiento de grano de maíz y las características y propiedades físico-químicas del suelo.

Identificar sistemas de labranza específicos para diferente suelos y cultivo de maíz.

\section{Hipótesis}

No existe diferencia en el rendimiento de grano de maíz bajo diferentes intensidades de labranza en un mismo suelo.

No existe diferencia en el rendimiento de grano de maíz en diferentes suelos bajo una misma intensidad de labranza.

Las características y propiedades físico-químicas de diferentes suelos no son afectadas por las intensidades de labranza y por lo tanto, estas no afectan el rendimiento de grano de maíz.

\section{MATERIALES Y MÉTODOS}

\section{Tratamientos estudiados}

En la zona central de Veracruz, México, bajo condiciones de temporal (estación lluviosa) en 1993 se evaluaron los siguientes sistemas de labranza:

1. Labranza convencional: este tratamiento consiste de un barbecho con arado de disco y dos pasos de rastra más surcado.

2. Labranza reducida: Consiste sólo de un barbecho y surcado.

3. Labranza cero: Sin movimiento de suelo durante toda la duración del experimento.

4. Labranza cero en rotación: Igual que tres sin movimiento de suelo, pero según se presente o no, una disminución en los rendimientos debido a cambios en las características del suelo, se le aplicará alguna intensidad de labranza, para obtener un sistema de rotación de labranza.
Los tratamientos se establecieron simultáneamente en un suelo Vertisol de textura fina y mal drenado; en un Fluvisol de textura fina drenado; en un Fluvisol de textura media drenado, localizados en terrenos del Campo Experimental Cotaxtla del Instituto Nacional de Investigaciones Forestales y Agropecuarias (INIFAP) y en un Luvisol de textura gruesa muy drenado, localizado en el Campo Experimental La Posta del INIFAP. El tamaño de la parcela fue de 12,8 × 25 metros.

\section{Manejo de tratamientos y cultivo}

La preparación de la cama de siembra en labranza reducida y convencional se realizó con arado y rastra de discos y surcadora de tracción motriz. En cero labranza se utilizaron 3 1/ha de Gramoxone (Paraquat) diluídos en 300 1/ha de agua más el Surfactante no-iónico Agralplus en dosis de 2,25 1/ha $(75 \% \mathrm{vol} / \mathrm{vol})$. La aplicación del herbicida se realizó con bomba manual de mochila. Además de lo anterior, con el fin de simular en este primer ciclo el efecto del "mantillo", en los dos tratamientos de cero labranza se aplicaron residuos de cosecha de maíz que cubrieron entre el 50 y $60 \%$ de la superficie del suelo.

En todos los tratamientos se sembró la variedad VS536 y la siembra se realizó en la segunda semana de julio en forma manual con espeque, depositando tres semillas por "golpe" a una profundidad entre cuatro y seis centímetros, para después aclarear a dos matas por "golpe" a los 20 días después de la siembra (DDS).

La distancia entre golpes varió de 40 a $50 \mathrm{~cm}$ y la distancia entre surcos y/o hileras de maíz fue de $80 \mathrm{~cm}$. En los tratamientos de labranza reducida y convencional se sembró en el fondo del surco excepto en los suelos arcillosos mal drenados donde se sembró en el lomo del surco. Con lo anterior se estimaba una densidad de población apróximada de 57000 plantas/ha. Sin embargo, al momento de la cosecha ésta varió para cada tratamiento.

El control de malezas se realizó mediante cultivos mecánicos para los tratamientos de labranza reducida y con vencional, en cero labranza se utilizaron $3 \mathrm{l} / \mathrm{ha}$ de Gramoxone mezclados con 2 1/ha de Gesaprim (Atrazina). Los herbicidas se diluyeron en 375 1/ha de 
agua y se aplicaron con bomba manual de mochila entre los 3 y 4 DDS, pero antes de la emergencia del maíz.

Todas las parcelas se fertilizaron con la fórmula 13846-00 kg/ha de NPK respectivamente. Todo el fósforo y el 50\% del nitrógeno se aplicaron entre los 15 y 20 DDS y el 50\% restante del nitrógeno entre los 45 y 50 DDS. Las fuentes de fertilizante usadas fueron la Urea (46\%) y el Superfosfato Triple de Calcio (46\%). Los fertilizantes se aplicaron en forma mateada y superficial sin enterrarse

Durante el desarrollo del cultivo se presentó el gusano Cogollero (Spodoptera frugiperda) y para su control se realizaron dos aplicaciones de Sevín $80 \%$ en dosis de $1,2 \mathrm{~kg} / \mathrm{ha}$ y una aplicación de Lorsban 480 en dósis de 0,35 l/ha.

Una vez que el maíz llegó a su madurez fisiológica a los 90 DDS se dobló para que se secara el grano y se cosechó entre los 130 y 140 DDS.

\section{Variables medidas}

Las variables medidas fueron la densidad de población al momento de la cosecha y el rendimiento de grano. Además, se registró la precipitación pluvial durante el ciclo del cultivo y se calculó [a relación Beneficio/Costo para cada sistema de labranza en cada tipo de suelo.

En este punto es necesario mencionar que además de las variables especificadas, en el experimento se contempla medir varias variables físicas y químicas del suelo que se relacionan con la intensidad de labranza y el rendimiento de grano de maíz. La medición de estas variables se iniciará a partir del segundo ciclo de la evaluación.

\section{RESULTADOS Y DISCUSIÓN}

\section{Densidad de población}

En el Cuadro 1 se muestra la densidad de población al momento de la cosecha para cada sistema de labranza en los cuatro tipos de suelo.

Como se observa en este cuadro, en los suelos fluvisoles de textura media y fina drenados, se obtuvo la mayor y más uniforme densidad de población con las tres intensidades de labranza. En el suelo luvisol arenoso muy drenado, la densidad de población fue mayor en cero labranza que en la labranza reducida y convencional y viceversa en el suelo vertisol arcilloso mal drenado.

Esta variación es la densidad de población al momento de la cosecha en los diferentes tipos de suelo puede ser explicado en gran parte por el grado de drenaje del suelo y la lámina y distribución de la precipitación pluvial ocurrida antes de la siembra y durante los primeros 40 días del cultivo principalmente.

Como se observa en la Figura 1, en el Campo Experimental Cotaxtla se presentarán altos volúmenes de lluvia antes de la siembra y durante el período de 10 a 40 días después de ésta. Esta situación originó que en el suelo vertisol mal drenado se presentara un considerable

Cuadro 1. Densidad de población de maíz (plantas/ha) al momento de la cosecha en cuatro suelos y cuatro intensidades de labranza. PV-1993.

\begin{tabular}{lcccc}
\hline Tratamiento & $\begin{array}{c}\text { Vertisol fino } \\
\text { mal drenado }\end{array}$ & $\begin{array}{c}\text { Fluvisol } \\
\text { fino drenado }\end{array}$ & $\begin{array}{c}\text { Fluvisol } \\
\text { medio drenado }\end{array}$ & $\begin{array}{c}\text { Luvisol grueso } \\
\text { muy drenado }\end{array}$ \\
\hline Labranza convencional & 54000 & 53000 & 56000 & 37500 \\
Labranza reducida & 41000 & 52000 & 57000 & 43125 \\
Labranza cero & 17500 & 55500 & 56500 & 51250 \\
Labranza cero en rotación & 17000 & 55000 & 56500 & 41875 \\
\hline
\end{tabular}




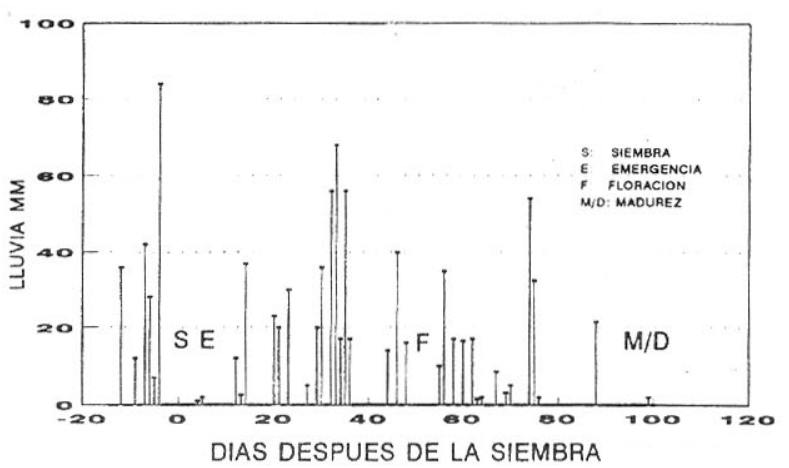

Fig. 1. Precipitación pluvial en el C. E. Cotaxtla. Ciclo PV-1993.

exceso de humedad durante el período de crecimiento, resultando en una alta mortandad y pobre desarrollo de plantas jóvenes, principalmente bajo el sistema de cero labranza. En la labranza convencional y labranza reducida la mayor densidad de población se debió en gran parte al hecho de sembrar en el lomo del surco, permitiendo a la planta "escapar" del exceso de humedad, aunque su crecimiento y desarrollo fue menor que en los otros suelos bajo estos mismos sistemas de labranza.

En los suelos fluvisoles de textura fina y media drenados, se presentó la misma lámina y distribución de la precipitación pluvial (Figura 1), sin embargo, debido a su mayor capacidad de drenaje no se observó exceso de humedad por períodos prolongados de tiempo como sucedió en el suelo vertisol, por lo que la mortandad de plantas fue prácticamente nula, observándose en los cuatro sistemas de labranza un buen crecimiento y desarrollo de plantas.

En la Figura 2 se muestra que en el suelo luvisol de textura gruesa muy drenado, localizado en el Campo Experimental La Posta, se presentaron altos volúmenes de precipitación pluvial antes de la siembra y entre los 20 y 35 días después de ésta, pero no se observó exceso de humedad.

Sin embargo, para este suelo con baja capacidad de retención de humedad, es más relevante el período de

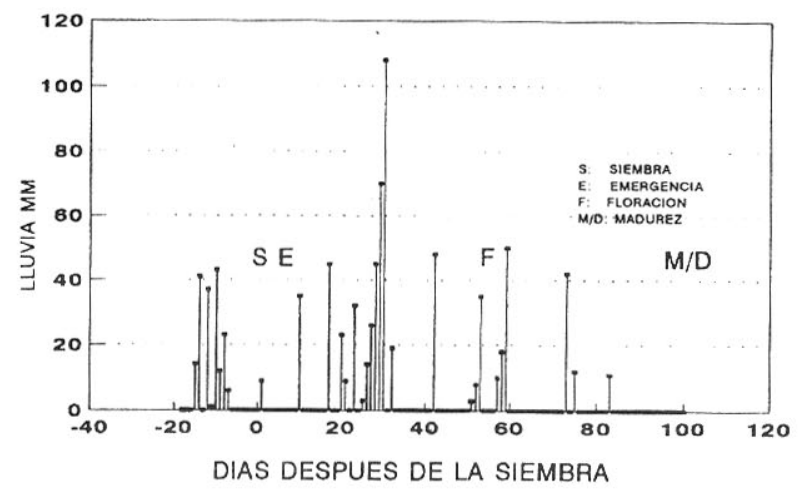

Fig. 2. Precipitación pluvial en el C. E. La Posta. Ciclo PV-1993.

quince días sin lluvia que se presentóinmediatamente antes y después de la siembra.

Probablemente se debe a este período de sequía que se redujo la densidad de población en los tratamientos de labranza convencional y labranza reducida, ya que aunque no se realizó un conteo de plantas jóvenes, si se observó en estas dos parcelas una menor densidad de población y en labranza convencional un menor crecimiento y desarrollo de plantas. Para la parcela de labranza cero en rotación no se cuenta con observaciones que permitan explicar en forma satisfactoria su reducción en la densidad de población, pero si se puede indicar que el crecimiento y desarrollo del maíz fue similar al de su parcela homóloga de labranza cero.

En resumen, podemos decir que en este primer año del experimento el alto volumen de precipitación pluvial antes de la siembra y durante los primeros 40 días del cultivo originó exceso de humedad en el suelo vertisol mal drenado reduciendo considerablemente la densidad de población en labranza cero. En los suelos fluvisoles de textura fina y media no se presentó tal exceso de humedad por lo que la densidad de plantas no se vió afectada. En el suelo luvisol de textura gruesa el período de sequía que se presentó antes y después de la siembra redujo la densidad de población en la labranza convencional y reducida. 


\section{Rendimiento de grano}

En el Cuadro 2 se muestra el rendimiento de grano obtenido con los cuatro sistemas de labranza en los cuatro tipos de suelo.

Como se observa en este cuadro, los mayores rendimientos se obtuvieron en los suelos fluvisoles de textura fina y media drenados, no existiendo practica mente diferencia entre éstos, ni entre los cuatro sistemas de labranza. De acuerdo con ésto, se puede decir que en estos suelos fluvisoles con alto potencial productivo y sin limitantes de exceso o falta de humedad durante las etapas críticas del cultivo, se puede obtener una alta y uniforme densidad de población así como altos rendimientos de grano, independientemente del sistema de labranza que se use.

En el suelo ]uvisol de textura gruesa muy drenado el rendimiento de grano fue significativamente menor que en los fluvisoles con los cuatro sistemas de labranza, y además los rendimientos obtenidos con los sistemas de labranza reducida y labranza cero son prácticamente iguales entre sí, pero superiores al rendimiento obtenido con el sistema de labranza convencional. Estos menores rendimientos con los cuatro sistemas de labranza se deben en parte a la baja fertilidad y menor capacidad de retención de humedad de estos suelos arenosos, lo cual pudo ser crítico durante las etapas de antes y después de ]a floración, cuando se presentó el menor volumen de precipitación pluvial (Figura 2). La diferencia en el rendimiento entre los cuatro sistemas de labranza en este suelo se debe en parte a la diferencia en la densidad de población y principalmente a los sistemas de labranza. En efecto, Phillips (1974) y Phillips et al. (1980) indican que en los sistemas de labranza reducida y labranza cero se conserva mayor humedad disponible en el suelo y los cultivos son más eficientes en el uso del agua que en el sistema de labranza convencional, por lo tanto, es probable que bajo estos sistemas de labranza de conservación se conservó mayor humedad en el suelo lo cual resultó en un mayor rendimiento de grano.

En el suelo Vertisol de textura arcillosa mal drenado el rendimiento de grano fue significativamente menor al obtenido en los suelos fluvisoles y luvisoles con los cuatro sistemas de labranza. Además, también se observa que en este suelo el rendimiento de grano bajo los sistemas de labranza convencional y reducida fue superior al obtenido con el sistema de labranza cero.

En estos suelos arcillosos mal drenados el exceso de humedad que prevaleció prácticamente durante todo el ciclo del cultivo (Figura 1) redujo drásticamente la densidad de población (principalmente en labranza cero) y el crecimiento y desarrollo del cultivo en los cuatro sistemas de labranza lo cual resultó en redimiento muy inferior a los obtenidos en los otros tipos de suelos. La diferencia en rendimiento entre los cuatro sistemas de labranza se relaciona con el exceso de humedad y la densidad de población como se explicó en el punto anterior.

De acuerdo con estos resultados se puede decir que para obtener los mejores rendimientos de grano de maíz, en los suelos fluvisoles de textura fina y media pero drenados se puede utilizar cualquiera de los sistemas de labranza estudiados, en cambio, en el suelo luvisol de

Cuadro 2. Rendimiento de grano de maíz ( $\mathrm{kg} / \mathrm{ha}$ ) obtenido con cuatro sistemas de labranza en cuatro tipos de suelo. PV-1993. (Humedad del grano: $12 \%$ ).

\begin{tabular}{lcccc}
\hline Tratamiento & $\begin{array}{c}\text { Vertisol fino } \\
\text { mal drenado }\end{array}$ & $\begin{array}{c}\text { Fluvisol } \\
\text { fino drenado }\end{array}$ & $\begin{array}{c}\text { Fluvisol } \\
\text { medio drenado }\end{array}$ & $\begin{array}{c}\text { Luvisol grueso } \\
\text { muy drenado }\end{array}$ \\
\hline Labranza convencional & 1854 & 4613 & 4898 & 2126 \\
Labranza reducida & 1110 & 4935 & 5346 & 4015 \\
Labranza cero & 662 & 5014 & 4807 & 3843 \\
Labranza cero en rotación & 751 & 4510 & 5045 & 3584 \\
\hline
\end{tabular}


textura gruesa muy drenado, es necesario utilizar un sistema de labranza reducida o cero. En el suelo vertisol arcilloso mal drenado ninguno de los sistemas de labranza estudiados ofrece rendimientos aceptables a menos que se use un sistema de drenaje o manejo de humedad del suelo, que permita eliminar el efecto negativo del exceso de humedad.

Con estos resultados se cumplen para este primer ciclo de evaluación los objetivos planteados al principio de este trabajo así mismo, se puede decir que la primera hipotésis planteada se rechaza para los suelos vertisol arcilloso mal drenado y luvisol arenoso muy drenado, y se acepta para los suelos fluvisoles de textura fina y media drenados. En relación con la segunda hipotésis, esta se acepta sólo entre los suelos fluvisoles pero se rechaza al considerar los cuatro suelos estudiados. Para la tercera hipotésis no se cuenta aún con información para aceptar/a o rechazarla.

\section{Relación Beneficio/Costo (B/C)}

Con los precios promedio de insumos y mano de obra vigentes en la zona en el primer trimestre de 1994 y un precio de garantía del grano de maíz de 224.5 US dólares por tonelada ( $\mathrm{N} \$$ :US DLLS $=3,34: 1$ ), se calcularon para cada sistema de labranza en los cuatro tipos de suelo los costos totales de producción y beneficio bruto a partir de lo cual se calculó la relación Beneficio/Costo (B/C). En el Cuadro 3 se muestran los resultados obtenidos.

Como se observa en este cuadro en los suelos fluvisoles de textura fina y media drenados, se obtuvieron las mayores relaciones beneficio/costo seguidos por el suelo luvisol de textura gruesa muy drenado y vertisol de textura arcillosa mal drenado. Comparando los cuatro sistemas de labranza, se observa que, excepto en los vertisoles arcillosos mal drenados, bajo los sistemas de labranza cero y reducida se obtuvieron las mayores relaciones beneficio/costo, lo cual se debióprincipalmente a la reducción en los costos de producción e incremento en el rendimiento de grano, al comparar/os con los del sistema de labranza convencional. En el caso de labranza cero, la reducción en $25 \%$ de los costos de producción incrementaron los beneficios económicos, aún y cuando se obtuvieron rendimientos de grano ligeramente menores que en labranza reducida.

En el suelo vertisol de textura fina mal drenado, la relación $\mathrm{B} / \mathrm{C}$ menor que la obtenida con los cuatro sistemas de labranza, indica que en estos suelos cuando hay altas probabilidades de exceso de humedad, no es posible producir maíz económicamente con cualquier nivel de labranza.

\section{CONCLUSIONES}

En los suelos fluvisoles de textura fina y media drenados, los sistemas de labranza estudiados no presentaron efecto en el rendimiento de grano de maíz y por lo tanto, desde el punto de vista económico es mejor utilizar los sistemas de labranza cero o reducida.

En el suelo luvisol de textura gruesa muy drenado, se obtuvo mayor rendimiento de grano y beneficio económico con los sistemas de labranza cero y reducida que con el sistema de labranza convencional.

Cuadro 3. Relación Beneficio/Costo (B/C) para cada sistema de labranza y tipo de suelo estudiado.

PV1993.

\begin{tabular}{lcccc}
\hline Tratamiento & $\begin{array}{c}\text { Vertisol fino } \\
\text { mal drenado }\end{array}$ & $\begin{array}{c}\text { Fluvisol } \\
\text { fino drenado }\end{array}$ & $\begin{array}{c}\text { Fluvisol } \\
\text { medio drenado }\end{array}$ & $\begin{array}{c}\text { Luvisol grueso } \\
\text { muy drenado }\end{array}$ \\
\hline Labranza convencional & 0,62 & 1,54 & 1,63 & 0,71 \\
Labranza reducida & 0,41 & 1,81 & 1,96 & 1,47 \\
Labranza cero & 0,2 & 2,20 & 2,10 & 1,68 \\
Labranza cero en rotación & 0,33 & 1,97 & 2,20 & 1,57 \\
\hline
\end{tabular}


En el suelo vertisol de textura arcillosa mal drenado, el rendimiento de grano fué muy inferior al obtenido en los otros suelos debido al exceso de humedad, y a menos que se mejore el drenaje del suelo, no es posible producir maíz en forma económica con cualquiera de los sistemas de labranza estudiados.

Considerando sólo las variables de densidad de población, rendimiento de grano y costos de producción, se probó que es necesario identificar el sistema de labranza más adecuado para producir maíz en diferentes tipos de suelo.

\section{LITERATURA CITADA}

COSPER, H.R 1983. Soil suitability for conservation tillage. Journal of Soil and Water Conservation. May-June: 152155 .

FRANZEN, H.; LAL, R.; EHLERS, W. 1994. Tillage and mulching effect on physical properties of a tropical alfisol. Soil and Tillage Research. 28: 329-346.

PHILLIPS, R.E. 1974. Soil water evapotranspiration and soil temperature in no-tilled soil. No-tillage research conference proceedings. University of Kentucky. Kentocky, USA. p. 6-15.

PHILLIPS, RE.; BLEVINS, R,L.; THOMAS, G.W.; FRYE, W. W.; PH!LLIPS. S.H. 1980. No-tillage agricultore. Science 208: 1108-1113.
RHOTON, F.E.; BRUCE, RR.; BUEHVING, N.W.; ELKINS, G.B.; LANGDALE, C.W.; TYLER, D.D. 1993. Chemical and physical characteristics off four soil types under convertional and no-tillage systems. Soil and tillage Research. 28:51-61.

URESTI, G.J.; CORNISH, G.A. 1986. Investigación inicial sobre la conservación de suelos en México. In: Memoria de la conferencia sobre la formación de una Red de Mecanización Agrícola para el pequeño agricultor. Campo Exp. Cotaxtla. Veracruz, Ver. México. p. 331-345.

URESTI, G.J.; CADENA, Z.M. 1994. Eficiencia de tres prácticas para conservar el suelo y su productividad en la zona tropical del centro de Veracruz. Revista INIFAP AgricoltoraTécnicaenMéxico. (En Prensa).

VYN, T.S.; DAYNARD, T.B.; KETCHESON, 1.W. 1982. Effect of reduced tillage systems on soil physical properties and maize grain yield in Ontario. In: Proceedings of the 9th Conference of the international Soil Tillage Research Organization (ISTRO). Socialistic Federal Republic of Yugoslavia. OSIJEK, 1982. P. 156-161.

WENDT, Re.; BURWELL, RE. . 1985. Runoff and soil losses for conventional, reduced and no-till corno Journal of SoiJ and Water Conservation. 40: 450-454. 